\title{
D-ZERO CRYOSTAT SUPPLEMENTAL RUPTURE DISC
}

\author{
G. T. Mulholland
}

\section{$8 / 3 / 87$}

D-Zero Engineering note 3740.000-EN-101 


\section{Introduction}

The common relief and rupture disc vent line requires o double disc assembly with vented interspace for occurote disc burst pressures. The first disc must toke pump and purge vacuum looding, but be set to operate of 1108 of the MAWP, 18.3 psig (ASME code). The ovoiloble solution is $18.3 \mathrm{psig}$ with o burst tolerance of $+/-2 \mathrm{psig}$. The interspace should be locally vented by oflow limiting vent valve to decouple the vent line bockpressure from the vessel rupture disc. The second disc must take the worst cose vent line backpressure, the steady stote value found in D-Zero engineering note $3740.000-E N-63$ with all three cryostots simultaneously venting of the fire condition into the $4^{-} \times 6^{-1}$ and $6^{\prime \prime} \times 0^{-1}$ sections. This value is less than 2 psid.

The moximum rupture volue for the second disc must be less than the minimum rupture volue for the first disc less 2 psid i.e. $<16.3-(+$ tolerance, see quotation).

\section{Sizing}

The minimum disc size required by the fire flow condition is $2^{-}$ pipe, but $3^{-}$is required by the suppliers of these low burst pressures. Normal monufocturing tolerances ore -108 to +208 typically. Speciol controls norrow the normal manufocturing tolerances by $758,50 \%$ and $25 \%$ ot on optional, but not prohibitive, cost. An effort to minimize manuf acturing tolerances has been made.

\section{Double Rupture Disc Specification}

Assembly (Final)

Number of complete ossemblies

Total number of discs (2 types)

Pipe Size

Double disc, insert

Interspoce flow limit valve

Interspoce pressure goge
4 ( 3 installed, one spore)

6 each minimum

3"

No bolt holes

Vents indoors

Code required

\section{Operating Pressure}

Nominal

1.2 otm. (3 psig) 


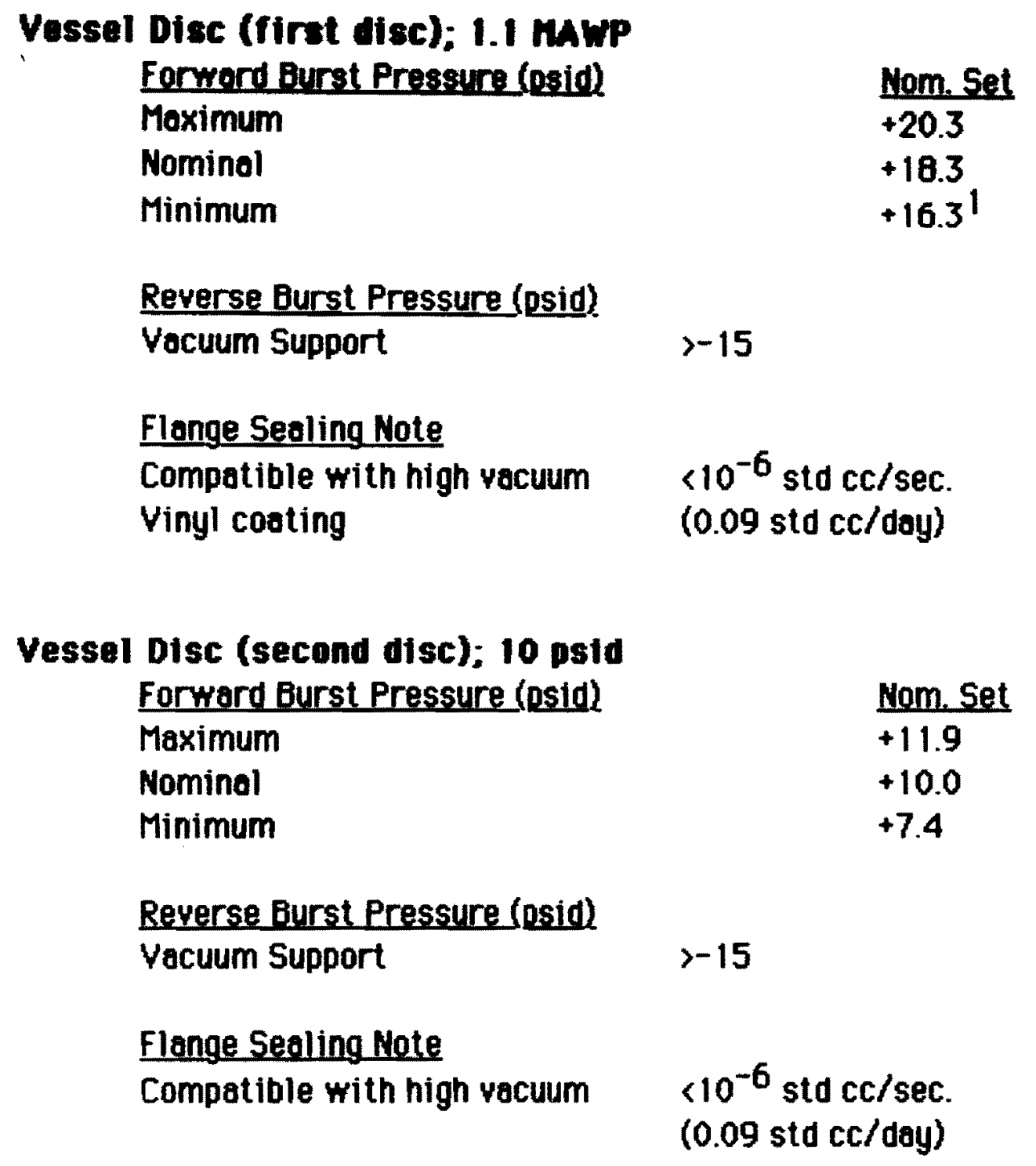

1. Implies a maximum relief valve set pressure < $13.2 \mathrm{psig}$ setting or 16.3 psig minimum setting to assure the relief valve is flowing fully before the rupture disc bursts. The ordered relief has a set pressure of 13 psig. 


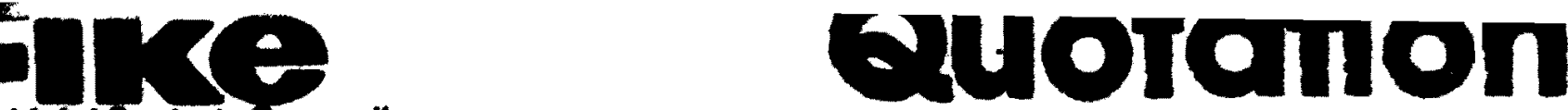

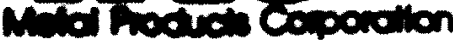

704 South 10 h Street - P.O. Box 610 - Blue Springs. Missouri 0.015 - (816) 229-3405 - Telex 42263

Ju1y 15, 19987

ermi

P O Box 500

Batavia, IL c/0 The Drager Company

415 Bant Goll Road - sulte 114

Arlington Beighta, IL 60005

Telephone: $312 / 640-1180$

Subject: Our Quote: CB-FMPD-87129

Attention: Mr. George Mulholland, as 357 x4591

\begin{tabular}{|c|c|c|c|c|c|c|c|c|}
\hline & \multirow{2}{*}{\multicolumn{3}{|c|}{$\begin{array}{l}\text { HOOOEA SPECIFICATION } \\
\text { Mominal Stas }\end{array}$}} & Iten 1 & Iten 2 & $\operatorname{Itan} 3$ & Iten 4 & \\
\hline 1 & & & & 3" & & & & \\
\hline 2 & \multirow{2}{*}{\multicolumn{3}{|c|}{$\frac{\text { Ration }}{\text { Aceomoly ype }}$}} & 150 & & & & 2 \\
\hline 3 & & & & Double $G$ & & & & 3 \\
\hline 4 & \multicolumn{3}{|c|}{ Drawing Number } & Insert Type & & & & 4 \\
\hline 5 & \multirow{3}{*}{ Material } & \multicolumn{2}{|c|}{ Bane } & 304 & & & & \\
\hline 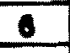 & & \multirow{2}{*}{\multicolumn{2}{|c|}{\begin{tabular}{|l} 
Holdodown \\
3rd. Comp
\end{tabular}}} & 304 & & & & 8 \\
\hline 7 & & & & 304 & & & & 7 \\
\hline 8 & \multicolumn{3}{|c|}{ Accensorios } & $1 / 4^{\prime \prime}$ gauge $t$ & bo & & & 8 \\
\hline \multicolumn{9}{|c|}{ ercess flou } \\
\hline 10 & & & & value, alloy & & & & 10 \\
\hline \multirow{3}{*}{$\frac{11}{12}$} & & & & steel studs & F nuts for & & Alternate & 11 \\
\hline & & & & Tfive flange & Ehicknesses. & Dounstream & Downstream & 12 \\
\hline & \multicolumn{3}{|c|}{ RUPTURE DISC SPECIFICATION } & & Dise & & Dles & \\
\hline 13 & \multicolumn{3}{|c|}{ Nominal Sko } & & 3" & 3⿻ & 3"- & 13 \\
\hline 14 & \multirow{2}{*}{\multicolumn{3}{|c|}{ Drawing Number }} & & $\mathrm{CPY}-\mathrm{BT}$ & CPY $-\mathrm{BX}$ & CPY $-8 R$ & 14 \\
\hline 15 & & & & & & & & \\
\hline - & \multirow{2}{*}{\multicolumn{3}{|c|}{$\begin{array}{l}\text { Specifind } \\
\text { Burst Precesure }\end{array}$}} & & 18 PSIG & to psraminal & L8 PSTG max. & 16 \\
\hline & & & & & & & & 17 \\
\hline $10^{-}$ & \multirow{2}{*}{\multicolumn{3}{|c|}{$\begin{array}{l}\text { Manufecturing } \\
\text { Panpe }\end{array}$}} & & Eero manf. & $8-11$ esIG & zaro manf. & 18 \\
\hline 19 & & & & & range. & & & 19 \\
\hline 20 & \multicolumn{3}{|c|}{ Aupture Folerance } & & $t=2,0 \mathrm{esIS}$ & & $+1-2,0$ esta & 20 \\
\hline 21 & \multirow{2}{*}{\multicolumn{3}{|c|}{ Spectifled Colncident Trmp }} & & $72^{\circ} \mathrm{p}$ & $22^{\circ}$ & 72 & 21 \\
\hline 2 & & & & & 316 SST & 316 SST YIR & 316 SST YIR & 22 \\
\hline 2 & \multirow{3}{*}{ moterial } & son & & & II & $\mathbf{M}$ & $M$ & 23 \\
\hline 24 & & $\sqrt{\operatorname{ver}}$ & m Support & & 316 SST VIR & 316 sst & 316 ssx & 24 \\
\hline \multirow[t]{2}{*}{25} & \multirow{2}{*}{\multicolumn{3}{|c|}{$\begin{array}{l}\text { ASME Burnt Contifications } \\
\text { PAICES }\end{array}$}} & & & & & 7 \\
\hline & & & & & & & & \\
\hline 20 & \multirow{2}{*}{\multicolumn{3}{|c|}{ 2-holders }} & 5605,10 & & & & 20 \\
\hline$\frac{2}{2}$ & & & & & & & & $\pi$ \\
\hline 2 & \multicolumn{3}{|c|}{6 -discs } & & $\$ 96.72$ & $\$ 74.40$ & $\$ 96.72$ & 28 \\
\hline \multirow{2}{*}{$\frac{29}{30}$} & & & & & & & & 29 \\
\hline & & & & & & & & 30 \\
\hline 31 & \multicolumn{3}{|c|}{ Attached condition } & f sal & shippins & 5 ueeks $A$ & ko. & 31 \\
\hline
\end{tabular}

nOTB 1: The "Downstream Disc" includes a vinyl costing on both sides of the "top ring" to minimize lealkage across the disc's to atmosphere via the excess flov valve located in the center section. should some leakage be acceptable, these vinyl coatings could

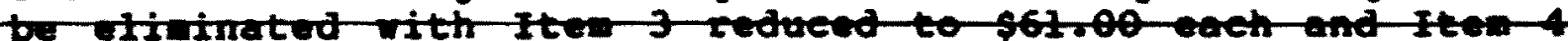

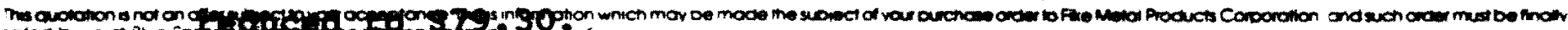

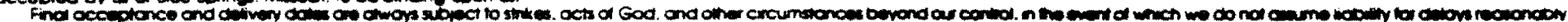
de to tech ocamence.

Terms of payment: 1810 days, net 30 days.

FIKE METAL PRODUCTA CORPORATION c/o Theprager coppany

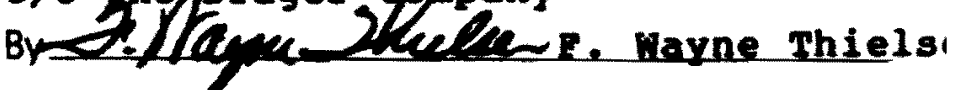

All shipments F.O.B. Blue Springs. Missouri.

uniess otherwise specified. 